\title{
"A ball in a cage"
}

\section{Răzvan O. Mada ${ }^{1}$, Raluca Tomoaia ${ }^{1}$, Vasile Sasarman ${ }^{2}$, Svetlana Encica ${ }^{3}$, Dan Bindea ${ }^{2}$}

${ }^{1}$ Department of Cardiology, ${ }^{2}$ Department of Cardiac Surgery, ${ }^{3}$ Department of Pathology, "Niculae Stancioiu” Heart Institute, Cluj-Napoca, Romania

\section{To the Editor,}

A 72-year-old female with a history of stroke and hypertension, without anticoagulant therapy, was referred for paroxysmal breathlessness. Clinical examination revealed irregular heart sounds. ECG confirmed the suspicion of atrial fibrillation with $\mathrm{AV}=90 \mathrm{bpm}$. Laboratory tests were in the normal range. The echocardiography found a dilated left atrium with a ball-like mobile tumor (fig 1a), without attachment to the interatrial septum, intermittently obstructing the mitral orifice (fig 1b). After excluding a coronary heart disease (no coronary lesions on invasive angiography), the patient underwent imme- diate cardiac surgery with atrial tumor removal (fig 1c). The histopathological exam confirmed its thrombotic nature (fig 1d). One week later the patient was discharged in good clinical condition with oral anticoagulant therapy.

Atrial thrombosis is a usual finding in patients with atrial fibrillation and no anticoagulation [1,2]. Nevertheless, large thrombi, particularly with such a perfect ball shape are very uncommon. This shape is most likely due to the "Brownian motion" of the thrombus inside the dilated atrium. However, highly mobile thrombi do usually embolize into the systemic circulation shortly after their formation [2]. In this particular case, it remained captive into the left atrium and grew to almost

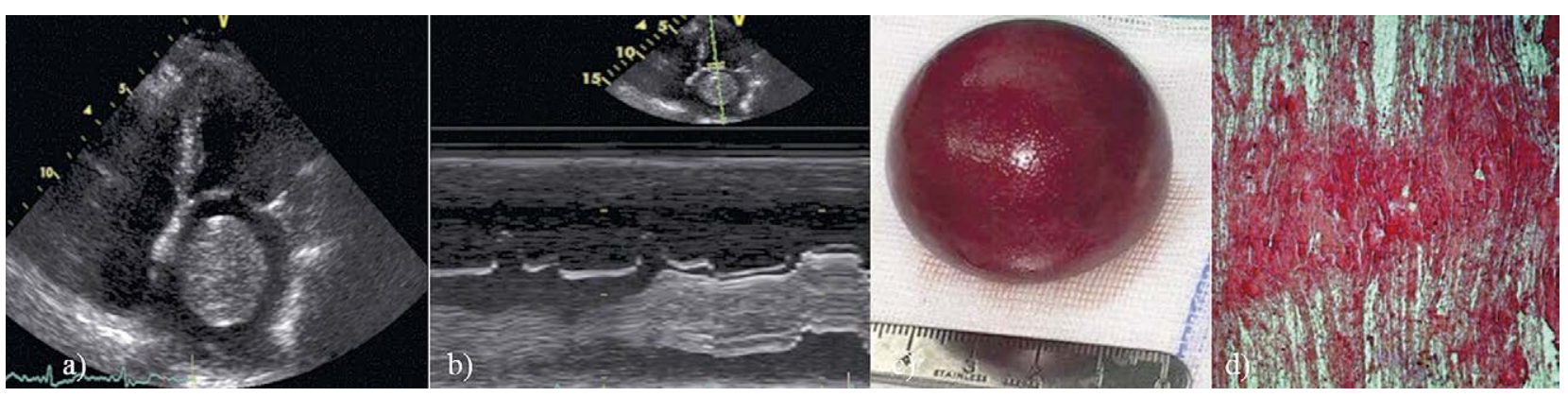

Fig 1. a) Echocardiography, apical four chambers view: mobile ball like tumor inside a dilated left atrium; b) Echocardiography, apical four chambers view: M-mode through the mitral valve showing intermittent obstruction of the mitral orifice by the tumor; c) Surgical specimen; d) Histopathological examination (microscopy): thrombotic fragment (fibrin network with very few blood cells and macrophages)

Received 10.10.2018 Accepted 29.10.2018

Med Ultrason

2018, Vol. 20, No 4, 539-540, DOI: 10.11152/mu-1752

Corresponding author: Dr Dan Bindea

"N Stancioiu" Heart Institute,

Department of Cardiac Surgery

Motilor 19-21, 400001, Cluj-Napoca, Romania

Phone: $+40 / 748121734$

E-mail: bindea_dan_ch@yahoo.com
$5 \mathrm{~cm}$, with a perfectly round and smooth shape. The large thrombus size hampered any further embolization as one piece, while fragmentation was unlikely considering its shape and consistency. Nevertheless, the intermittent obstruction of the mitral orifice had the potential to induce cardiac arrest at any time hence the reason why the situation called for immediate surgical therapy $[3,4]$. 


\section{References}

1. Kirchhof P, Benussi S, Kotecha D, et al. 2016 ESC Guidelines for the management of atrial fibrillation developed in collaboration with EACTS. Eur J Cardiothorac Surg 2016;50:e1-e88.

2. Violi F, Pastori D, Pignatelli P. Mechanisms And Management Of Thrombo-Embolism In Atrial Fibrillation. J Atr Fibrillation 2014;7:1112.
3. Blanche C, Chaux A, Kass RM, Helfenstein J, Sugarman G. Free-floating ball thrombus in the left atrium after mitral valve replacement: successful surgical management. Ann Thorac Surg 1985;39:566-568.

4. Wrisley D, Giambartolomei A, Lee I, Brownlee W. Left atrial ball thrombus: review of clinical and echocardiographic manifestations with suggestions for management. Am Heart J 1991;121:1784-1790.

\title{
Extra-tunnel compression mimicking tarsal tunnel syndrome: ultrasound imaging for a plantar ganglion cyst
}

\author{
Ke-Vin Chang ${ }^{1}$, Wei-Ting Wu ${ }^{1}$, Levent Özçakar ${ }^{2}$
}

${ }^{1}$ Department of Physical Medicine and Rehabilitation and Community and Geriatric Research Center, National Taiwan University Hospital, Bei-Hu Branch and National Taiwan University College of Medicine, Taipei, Taiwan, ${ }^{2}$ Department of Physical and Rehabilitation Medicine, Hacettepe University Medical School, Ankara, Turkey

\section{To the Editor,}

A 43-year-old man presented with a six-month history of right-foot numbness, which worsened after walking. Lumbar traction (applied for a suspected diagnosis of lumbosacral radiculopathy) was not beneficial. Initial ultrasound (US) examination under the tentative diagnosis of tarsal tunnel syndrome (TTS) did not identify any remarkable findings at the level of the medial malleolus. Owing to the failure of conservative treatments, he was referred for US-guided injection of the tibial nerve. Before the intervention, we tracked the tibial nerve from the posterior knee to the plantar area. No abnormality was identified proximal to the inferior border of the ankle flexor retinaculum (Fig 1A). Distally, when we attempted to move the transducer toward the plantar surface, the patient described aggravation of numbness. A dumbbellshaped anechoic well-demarcated mass was observed on top of the tibial nerve, in proximity to the medial and

Received 08.11.2018 Accepted 12.11.2018

Med Ultrason

2018, Vol. 20, No 4, 540-541 DOI: 10.11152/mu-1788

Corresponding author: Ke-Vin Chang, $\mathrm{MD}, \mathrm{PhD}$

Department of Physical Medicine and

Rehabilitation, National Taiwan University

Hospital, Bei-Hu Branch, No. 87 Neijiang St,

Wanhua District, Taipei City 108, Taiwan

E-mail: kvchang011@gmail.com; pattap@pchome.com.tw lateral plantar branches (Fig 1B, Video 1, on the journal site). The lesion was not enhanced under power Doppler imaging (Fig 1C). No similar pathology was seen in the contralateral ankle (Fig 1D). A ganglion cyst was diagnosed and his symptom disappeared after surgical excision.

The tarsal tunnel is defined by the flexor retinaculum (as its roof) and underlying tibia, talus, and calcaneus bones (as its floor) and comprises the tibialis posterior, flexor digitorum longus and flexor hallucis longus tendons, tibial nerve, and posterior tibial vessels. Enlargement/ thickening of this complex leads to increased intra-compartmental pressure and tibial nerve compression. Other common causes are space-occupying lesions, such as ganglion cysts, bony fragments and neurogenic tumors $[1,2]$.

Our patient had typical TTS symptoms. However, the initial US omitted the ganglion cyst as scanning was limited to the tarsal tunnel. The knot of Henry (distal to the tarsal tunnel) is vulnerable to plantar nerve compression [3]. Here, the flexor digitorum longus and flexor hallucis longus tendons cross over. The proximal medial and lateral plantar nerves travel superficial to the intersection point, which may lead to entrapment neuropathy in frequent runners wearing ill-fitting shoes. In this case, both plantar nerves were compressed by the ganglion cyst, which provoked symptoms mimicking TTS. Furthermore, the worsening symptoms after walking might indicate that the compressive point was located in the plantar region rather than the retro-malleolus area. 


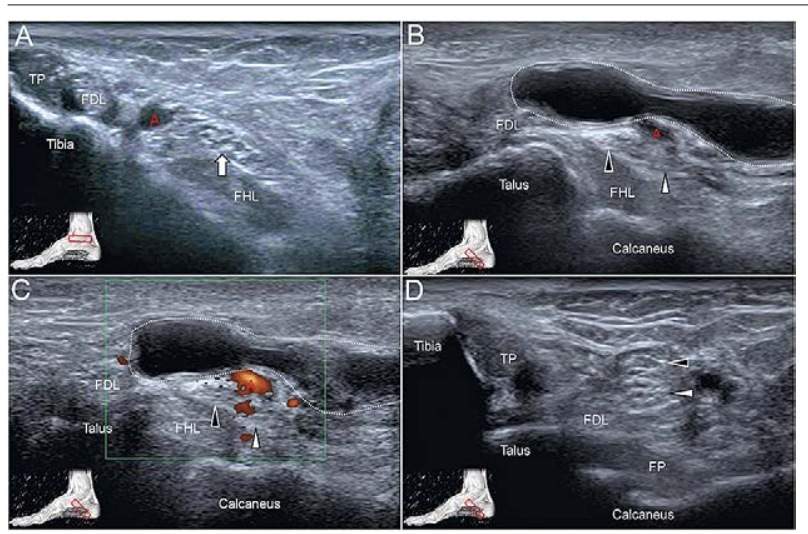

Fig 1. Ultrasound imaging of the retromalleolar (A) and plantar (B) regions of the medial aspect of the affected ankle; power Doppler imaging of the mass lesion (C); ultrasound of the asymptomatic ankle (D). White arrow: tibial nerve; black arrowhead, medial plantar nerve; white arrowhead, lateral plantar nerve; TP, tibialis posterior tendon; FDL, flexor digitorum longus tendon; FHL, flexor hallucis longus tendon; A, posterior tibial artery; dashed area, ganglion cyst.

Finally, power Doppler ultrasound should be routinely used for investigation of tibial (or plantar) nerve entrapment neuropathy. As shown in Figure 1B, the ganglion cyst looks similar to a dilated vessel or an aneurysm on grey-scale US imaging, which has been reported as a cause of TTS [4]. In conclusion, the present case highlights the importance of tracking the entire course of the affected nerve in peripheral nerve disorders and scout scanning prior to any perineural injections.

Acknowledgment: The present study is supported by (1) National Taiwan University Hospital, Bei-Hu Branch, (2) Ministry of Science and Technology (MOST 106-2314-B-002-180-MY3), and (3) Taiwan Society of Ultrasound in Medicine.

\section{References}

1. Wu WT, Chang KV, Ozcakar L. Ultrasound facilitates the diagnosis of tarsal tunnel syndrome: intraneural ganglion cyst of the tibial nerve. J Ultrasound 2018 Jul 17. doi:10.1007/s40477-018-0314-5.

2. Hong CH, Lee YK, Won SH, Lee DW, Moon SI, Kim WJ. Tarsal tunnel syndrome caused by an uncommon ossicle of the talus: A case report. Medicine 2018;97:e11008.

3. Beger O, Elvan O, Keskinbora M, Un B, Uzmansel D, Kurtoglu Z. Anatomy of Master Knot of Henry: A morphometric study on cadavers. Acta Orthop Traumatol Turc 2018;52:134-142.

4. Ayad M, Whisenhunt A, Hong E, et al. Posterior tibial vein aneurysm presenting as tarsal tunnel syndrome. Vascular 2015;23:322-326

\title{
Pyoderma gangrenosum as paraneoplastic syndrome in renal carcinoma
}

\author{
Ioana Duca, Flaviu Rusu, Nicolae Nicula, Dan Lucian Dumitrascu
}

$2^{\text {nd }}$ Internal Medicine Department, "Iuliu Hatieganu" University of Medicine and Pharmacy, Cluj-Napoca, Romania

\section{To the Editor,}

Paraneoplastic syndromes occur in about $8 \%$ of patients with cancer and may involve different organs and systems [1]. First described in 1930 by Brunsting et al [2]

Received 10.10.2018 Accepted 05.11.2018

Med Ultrason

2017, Vol. 19, No 4, 541-542, DOI: 10.11152/mu-1751

Corresponding author: Flaviu Rusu

$2^{\text {nd }}$ Internal Medicine Department, "Iuliu Hatieganu" University of Medicine and Pharmacy,

2-4 Clinicilor Street,

400006 Cluj-Napoca, Romania

E-mail: flaviurusu@yahoo.com pyoderma gangrenosum $(\mathrm{PG})$ is an idiopathic neutrophilic dermatosis that was once considered pathognomonic of idiopathic ulcerative colitis. It has since been described in association with a wide variety of disorders, including neoplasia: colon, bladder, prostate, breast, bronchus, ovary, adrenocortical carcinoma and solid tumors [3].

The association between renal carcinoma and PG is not commonly described in the literature; only 3 studies [4-6] have so far reported the association with renal cell carcinoma. Approximately $10-40 \%$ of patients with renal cancer develop paraneoplastic syndromes during their disease [7].

Recently, we had a 60-year-old patient admitted for dyspnoea, fever and long-lasting painful refractory ulcers 


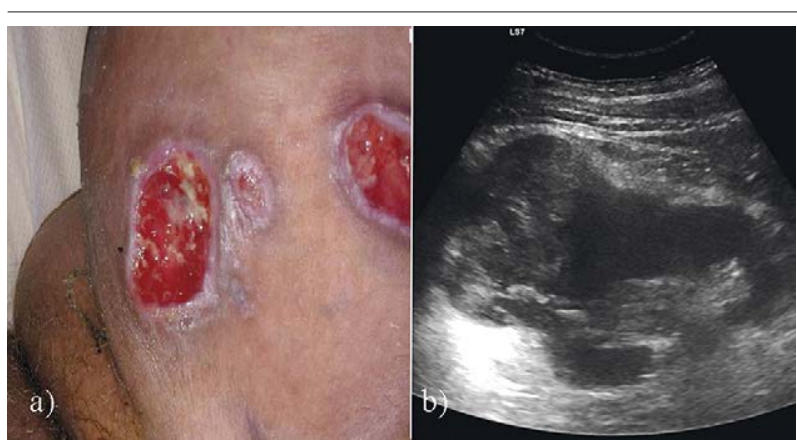

Fig 1. a) Clinical aspect of the right groin ulcerations; b) grayscale ultrasound showing the large tumor of the left kidney.

in the right groin (fig 1a). No associated macroscopic hematuria was present. General physical examination revealed painful ulceration of the right groin bordered by a reddish edge. Laboratory findings evidenced a high inflammatory syndrome and no microscopic hematuria or proteinuria. Bacteriological examination of inguinal ulcers showed Pseudomonas aeruginosa and Candida spp but no amelioration after the specific treatment was obtained. Histological examination demonstrated the presence of lymphocytic infiltrate, leukocytoclastic vasculitis and focal microabscesses, confirming the Pyoderma gangrenosum aspect. At abdominal ultrasound we found at the upper pole of the left kidney, a $7 \mathrm{~cm}$ inhomogeneous, imprecisely delimited and hypervascularizated solid mass, without invasion of the renal vein (fig 1b). CT staged the kidney tumor as T2aN0M0. Similar to other published cases [3], despite having positive cultures, the ulcers of our patient responded to corticosteroids instead of antibiotics.
The particularity of the case is the rarity of PG diagnosis as paraneoplastic syndrome in renal carcinoma and the lack of urinary syndromes.

The present case highlights the importance of tumor screening in patients with atypical antibiotic-refractory skin ulcers, even if there is no clinical evidence for an organ-specific neoplasia.

\section{References}

1. Baijens LW, Manni JJ. Paraneoplastic syndromes in patients with primary malignancies of the head and neck: four cases and a review of the literature. Eur Arch Otorhinolaryngol 2006;263:32-36.

2. Brunsting LA, Goeckerman WH, O'Leary PA. Pyoderma gangenosum: clinical and experimental observations in five cases occurring in adults. Arch Derm Syphilol 1930;22:655-680.

3. Crowson AN, Mihm MC Jr, Magro C. Pyoderma gangrenosum: a review. J Cutan Pathol 2003:30:97-107.

4. Regnier-Rosencher E, Bizet N, Mery L. Pyoderma gangrenosum associated with renal carcinoma. J Am Acad Dermatol 2011;64:1208-1211.

5. Cosgarea I, Lovric Z, Körber A, Dissemond J. Successfull treatment of refractory pyoderma gangrenosum with ustekinumab only after excision of renal cell carcinoma. Int Wound J 2016;13:1041-1042.

6. Solovan C, Smiszek R, Wickenhauser C, Chiticariu E. Postoperative Pyoderma gangrenosum in association with renal cell carcinoma and chronic lymphocytic leukemia. Infect Dis Ther 2013,2:7580.

7. Aldaabil R, Peereboom D. Paraneoplastic Syndromes in Renal Cell Carcinoma. In: Bukowski RM, Novick AC. (eds). Renal Cell Carcinoma. Current Clinical Oncology. Totowa, Humana Press 2000:135-145. 